\title{
Failure Analysis in FeCo Magnetic Alloys through Electron Channeling Contrast Imaging Defect Characterization
}

Julia Deitz ${ }^{1}$, Tim Ruggles ${ }^{2}$, Philip Noell ${ }^{1}$, Donald Susan ${ }^{3}$ and Joseph Michael ${ }^{1}$

${ }^{1}$ Sandia National Laboratory, United States, ${ }^{2}$ Sandia National Laboratories, Albuquerque, New Mexico, United States, ${ }^{3}$ Sandia National Laboratories, United States

FeCo based alloys have gained interest for applications such as solenoids, transformers, motors, and generators. This is because these alloys have demonstrated desirable magnetic properties with heat treatment. However, FeCo alloys tend to fracture readily, and the exact mechanisms behind this brittleness remain elusive. The brittle properties are thought to be related to the $\gamma$-FCC $\rightarrow \alpha$-BCC $\rightarrow \alpha_{2}\left(B_{2}\right)$ phase transformation that equiatomic or near-equiatomic Fe-Co alloys undergo when cooling from higher temperatures. It has been hypothesized that this process can lead to anti-phase boundaries in the ordered phase which could promote fracture. Here, we investigate structural defects in FeCo alloys and their role in fracture.

A heat-treated $\mathrm{Fe}-\mathrm{Co}-2 \mathrm{~V}$ alloy is investigated using electron channeling contrast imaging (ECCI), and this shows only specific grains towards the outer edges of the sample have defect content. These defects are shown in Figure 1(a) and appear consistent with anti-phase domains; if they exist, this would be indicative of ordering in the sample. These grains were further investigated with STEM shown in Figure 1(b) which show that these defects continue throughout the entire grain. HR-STEM and EDXS will be performed to conclusively determine the type of defect present in these grains. Only the grains with these defects show magnetic domains - shown with Lorentz TEM in Figure 2 - thus confirming the relationship between these defect structures and the magnetic properties of the FeCo alloy. In-situ strain ECCI will be shown to investigate the role these defects play in ultimate fracture. Understanding the role of structural defects in FeCo alloy fracture will help to mitigate their effects.

$\uparrow$ This paper describes objective technical results and analysis. Any subjective views or opinions that might be expressed in the paper do not necessarily represent the views of the U.S. Department of Energy or the United States Government. Sandia National Laboratories is a multimission laboratory managed and operated by National Technology and Engineering Solutions of Sandia, LLC., a wholly owned subsidiary of Honeywell International, Inc., for the U.S. Department of Energy's National Nuclear Security Administration under contract DE-NA-0003525. 

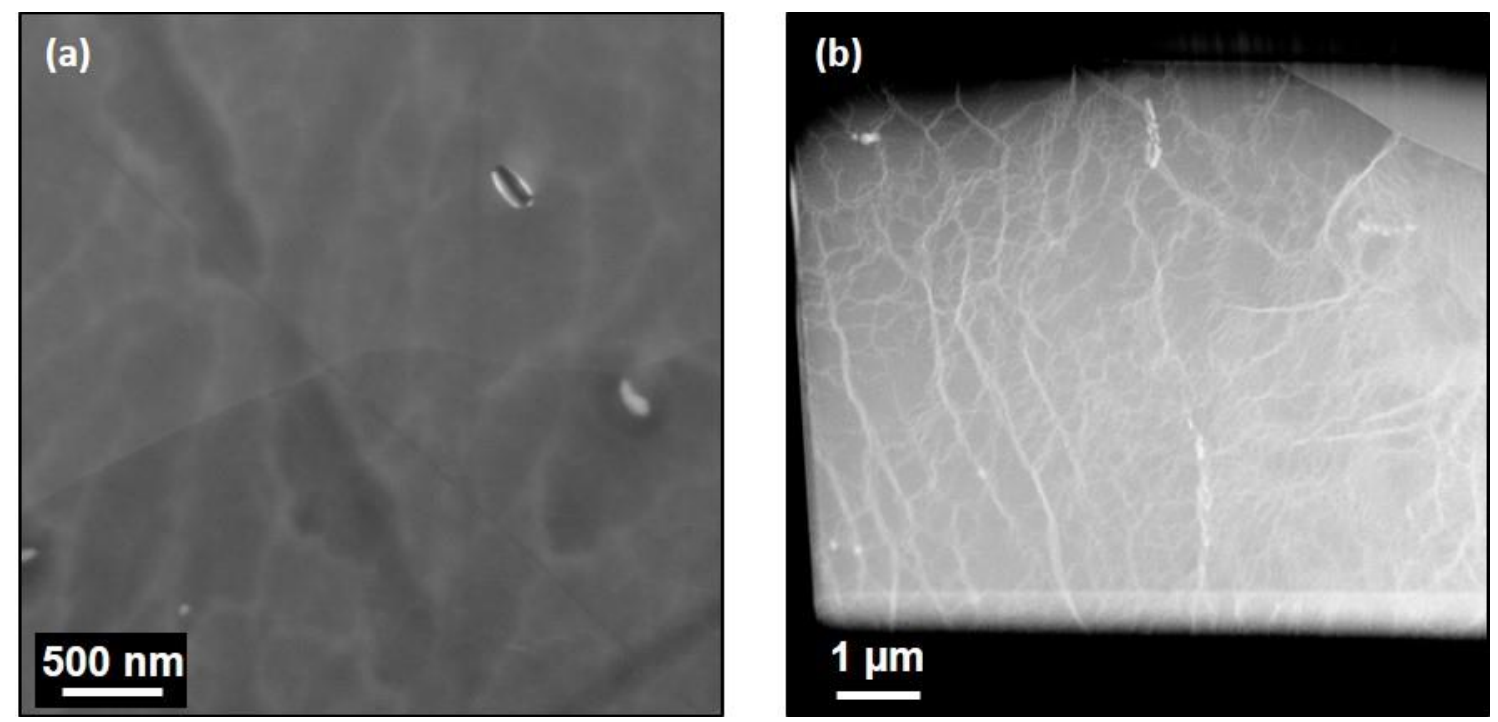

Figure 1. Figure 1. (a) ECCI image of a heat treated FeCo alloy sample showing structural defects and (b) correlated STEM image showing these defect structures continue throughout grain.

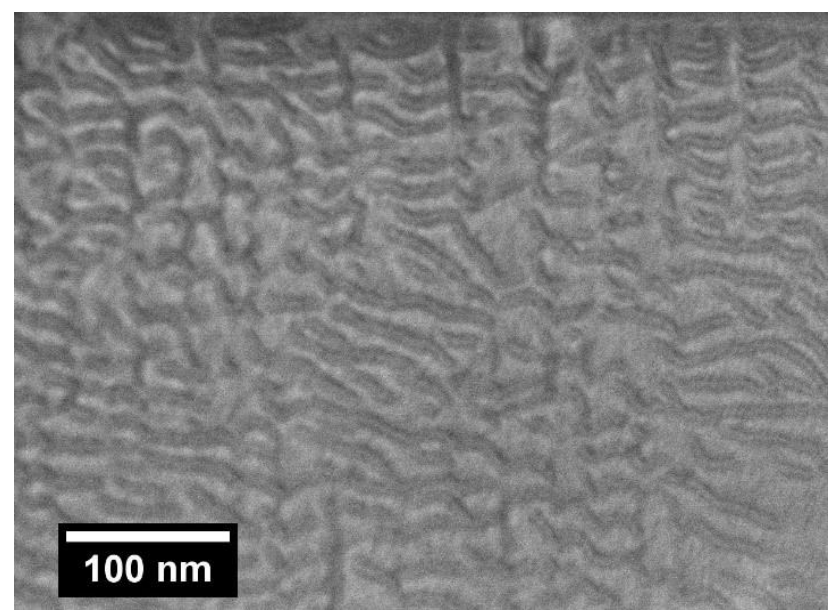

Figure 2. Figure 2. Magnetic domains in the sample shown in Figure 1(b). These are present only in the grains with structural defects. 